\title{
Influência e Impacto do Programa MPS.BR na Pesquisa Relacionada à Qualidade de Software no Brasil
}

\author{
Gleison Santos \\ Programa de Pós-Graduação em Informática - Universidade Federal do Estado do Rio \\ de Janeiro (UNIRIO) - Av. Pasteur, 458, Urca, CEP 22290-240 - Rio de Janeiro, RJ \\ gleison.santos@uniriotec.br
}

Resumo. O Programa Brasileiro de Melhoria de Processos de Software (MPS.BR) é uma iniciativa para melhorar a capacidade de desenvolvimento de software nas organizações brasileiras iniciada em 2003. O impacto do MPS.BR na indústria de software brasileira pode ser observado, por exemplo, a partir de indicadores de desempenho medidos (i.e., iMPS). No entanto, ainda não foram relatados estudos que procurem observar os impactos causados no ambiente de pesquisa de Qualidade de Software. Este artigo apresenta um estudo baseado em revisão sistemática da literatura que procura avaliar a influência e o impacto do MPS.BR na produção científica relacionada a Qualidade de Software no país.

\begin{abstract}
The MPS.BR, created in 2003, is an initiative to improve the Brazilian industry capacity to develop software. The positive impact of the MPS.BR can be observed, for instance, on performance indicators (e.g., iMPS). Nevertheless, there is a lack of studies describing the impact of MPS.BR in Software Quality research. This paper describes a study based on systematic literature review that shows the MPS.BR influence and its impact on the scientific research on Software Quality in Brazil.
\end{abstract}

\section{Introdução}

Acredita-se que o uso de boas práticas de Engenharia de Software possa melhorar o desempenho das organizações com respeito a custo, prazo, produtividade, qualidade, satisfação do cliente e retorno do investimento e, consequientemente, aumentar sua vantagem competitiva. O Programa Brasileiro de Melhoria de Processos de Software (MPS.BR) [SOFTEX 2009] é uma iniciativa sob a coordenação da Associação para Promoção da Excelência do Software Brasileiro (SOFTEX), com apoio do governo (MCT e FINEP), SEBRAE/PROIMPE e BID (Banco Interamericano de Desenvolvimento), da indústria de software brasileira e de instituições de pesquisa. A sigla MPS.BR está associada ao programa MPS.BR - Melhoria do Processo de Software Brasileiro e a sigla MPS está associada ao modelo MPS. O modelo MPS é composto pelo Modelo de Referência MR-MPS, Método de Avaliação MA-MPS e Modelo de Negócio MN-MPS.

O objetivo do MPS.BR é desenvolver e disseminar um modelo de melhoria de processos brasileiro (MR-MPS, Modelo de Referência MPS) visando estabelecer um caminho economicamente viável para que organizações, incluindo as pequenas e médias empresas (PME), alcancem os benefícios da melhoria de processos e da utilização de boas práticas da engenharia de software em um intervalo de tempo razoável [Kalinowski et al. 2010]. O programa MPS.BR tem duas metas: uma meta técnica, 
relativa à criação e evolução do Modelo MPS; uma meta de mercado, relativa à disseminação do modelo MPS tanto em PMEs quanto em grandes organizações públicas e privadas. No seu planejamento de longo prazo, o programa MPS.BR está alinhado ao objetivo da SOFTEX de desenvolvimento do mercado de software brasileiro e no aumento sustentável da competitividade da indústria brasileira de software e serviços [Santos et al. 2009].

O impacto do MPS.BR na indústria de software brasileira pode ser observado através do número de empresas já avaliadas no seu Modelo de Referência (MR-MPS) e, também, nos resultados quantitativos da adoção e disseminação do MPS e resultados qualitativos de desempenho das organizações que o adotaram [TRAVASSOS E KALINOWSKI 2011] [KALINOWSKI et al. 2008; 2010]. No entanto, ainda não houve relatos de estudos que procurem avaliar a influência e o impacto do MPS.BR na produção científica relacionada a Qualidade de Software no país.

Este artigo apresenta os resultados da execução de um estudo baseado em revisão sistemática da literatura para investigar a influência e o impacto do MPS.BR (e de seu modelo de referência) na pesquisa relacionada a processos de software no Brasil a partir da sua definição. A hipótese investigada é se o número de publicações científicas, sejam relatos de experiência ou trabalhos técnicos, que citam como aplicação ou justificativa o uso do MPS.BR/MR-MPS está crescendo no país. Ou seja, se há evidências de que a existência do modelo está fomentando a utilização de boas práticas de engenharia de software nas empresas e/ou se a existência do modelo influencia positivamente a descoberta de temas de pesquisa na academia que, posteriormente, espera-se que sejam aplicados na indústria.

Além desta introdução, este artigo está organizado em 4 seções. A Seção 2 descreve a metodologia utilizada para a condução da revisão sistemática, enquanto a Seção 3 apresenta detalhes de como este protocolo foi elaborado. A Seção 4 apresenta seus principais resultados. Por fim, as considerações finais são apresentadas na Seção 5.

\section{Protocolo do Estudo Baseado em Revisão Sistemática da Literatura}

A revisão sistemática é um tipo de estudo secundário [KITCHENHAM 2004], cujo processo de pesquisa segue um conjunto de passos metodologicamente bem definidos de acordo com um protocolo prévio [BIOLCHINI et al. 2005] e cuja adoção procura reduzir o viés inerente a uma revisão informal [SILVA FILHO 2006]. O desenvolvimento de uma abordagem sistemática de revisão visa estabelecer um processo formal para conduzir este tipo de investigação, evitando a introdução de eventuais vieses da revisão de literatura informal [MAFRA e TRAVASSOS 2006]. A estrutura do protocolo apresentado a seguir é baseada em [SANTOS 2008].

\subsection{Planejamento do Estudo}

Contexto: O MPS.BR é o programa para Melhoria de Processo do Software Brasileiro [SOFTEX 2009]. O impacto do MPS.BR na indústria de software brasileira pode ser observado através do número de empresas avaliadas, porém não há, ainda, relatos de estudos que procurem avaliar a influência e o impacto do MPS.BR na produção científica relacionada a Qualidade de Software no país.

Objetivo: Investigar a influência e o impacto do MPS.BR (e de seu modelo de referência) na pesquisa relacionada a processos de software no Brasil a partir da sua definição. Dessa forma, espera-se analisar relatos de experiência e publicações científicas por 
meio de um estudo baseado em revisão sistemática com o propósito de identificar a influência do MPS.BR com relação a pesquisa de qualidade de software do ponto de vista dos pesquisadores no contexto acadêmico e industrial.

Questão Principal: Foi formulada a seguinte questão principal de pesquisa:

QP 1. O Programa MPS.BR e o seu Modelo de Referência (MR-MPS) estão influenciando a pesquisa em Qualidade de Software no Brasil?

Questões Secundárias: As seguintes questões secundárias foram identificadas:

QS 1. Qual a relação entre a influência do MPS.BR e do CMMI na pesquisa em Qualidade de Software no Brasil?

QS 2. Além do MPS.BR e do CMMI, que outros métodos, normas, modelos e padrões de referência são citados pelas publicações selecionadas?

QS 3. Quando há citação ao MPS.BR e ao CMMI, é possível perceber se estes modelos servem como justificativa/fundamentação do trabalho?

QS 4. Quando há citação ao MPS.BR e ao CMMI, é possível perceber se a pesquisa descrita foi utilizada na prática (em trabalhos técnicos) ou estes modelos foram utilizados na prática nas organizações (em relatos de experiências)?

QS 5. Que processos, ou tipos de processos, do MR-MPS e do CMMI são citados?

QS 6. Há citação a avaliações MPS.BR e CMMI? Quais os níveis?

QS 7. Há evidências de que os métodos ágeis SCRUM e XP sejam citados em trabalhos (técnicos e relatos de experiência) relacionados a qualidade de software em organizações brasileiras em conjunto com o MR-MPS e o CMMI? Há evidencias de que fundamentem parte da pesquisa realizada e sejam aplicados na prática?

Intervenção: Uso do MPS.BR e do seu Modelo de Referência (MR-MPS).

Comparação: Não se aplica.

População: Artigos técnicos e relatos de experiência com foco em qualidade de software.

Resultados: A partir da identificação das citações e fundamentações de trabalhos científicos no MPS.BR, espera-se avaliar se a definição do modelo teve alguma influência no pesquisa em qualidade de software no país.

\subsection{Escopo da Pesquisa}

Para delinear o escopo da pesquisa foram estabelecidos critérios para garantir, de forma equilibrada, a viabilidade da execução (custo, esforço e tempo), acessibilidade aos dados e abrangência do estudo. A pesquisa dar-se-á a partir dos anais dos eventos a partir de consultas manuais e, se possível, de engenhos de busca, quando disponíveis.

Critérios adotados para seleção das fontes: Devem ser selecionados para consulta eventos patrocinados pela SBC (Sociedade Brasileira de Computação) cujo foco seja específico em Qualidade de Software. Os mecanismos de busca utilizados devem garantir resultados únicos através da busca de um mesmo conjunto de palavras-chaves. Quando isto não for possível, deve-se estudar e documentar uma forma de minimizar os potenciais efeitos colaterais desta limitação. Dentre dos eventos patrocinados pela SBC, o único que se encaixa nesta classificação é o SBQS (Simpósio Brasileiro de Qualidade de Software).

Restrições: A pesquisa está restrita à análise de publicações obtidas, exclusivamente, a 
partir das fontes selecionadas a partir dos critérios supracitados. O estudo englobará os dados disponíveis nas fontes considerando o período de 01 de janeiro de 2002 até 31 de dezembro de 2010.

Idiomas: Devem ser aceitos trabalhos escritos em português, inglês e espanhol, idiomas aceitos para publicação no SBQS.

\subsection{Métodos de Busca de Publicações}

Não há uma biblioteca digital que catalogue os anais do SBQS e que possibilite a pesquisa eletrônica. Dessa forma, todas as buscas serão manuais. Não será utilizado um conjunto de palavras chave para efetuar a busca pelos artigos. Todos os artigos publicados deverão ser analisados para decidir sobre sua inclusão ou não no estudo.

A identificação das palavras relevantes no conteúdo do artigo deve ser feita a partir da leitura dos títulos, campos de palavras-chave e resumos/abstracts (para identificar as palavras-chave) e texto completo, incluindo referências bibliográficas (para identificar as menções aos métodos, normas, modelos e padrões de referência citados).

Será utilizado um conjunto de palavras-chave para caracterização dos artigos, porém, estas palavras-chave serão apenas registradas, não sendo determinantes para a seleção ou não das publicações para o escopo deste estudo. As palavras-chave podem estar presentes em português, inglês e espanhol. As palavras-chave, em português, são: processo, processos, software, processo de software, processos de software, processo do software, desenvolvimento e manutenção. Para artigos em inglês e espanhol e abstracts, devem ser consideradas as traduções destas palavras-chave.

\subsection{Procedimentos de Seleção e Critérios}

A estratégia de busca será aplicada por um pesquisador para identificar as publicações em potencial. As publicações identificadas serão selecionadas pelos demais pesquisadores (incluindo o que fará a busca) através da verificação dos critérios de inclusão e exclusão e de qualidade estabelecidos. Os pesquisadores deverão entrar em consenso sobre a seleção das publicações cujas avaliações se mostrem conflitantes.

Em caso de impasse entre os pesquisadores, a publicação deverá ser incluída na lista de selecionadas. Para diminuir o risco que uma publicação seja excluída prematuramente em uma das etapas do estudo, sempre que existir dúvida a publicação não deverá ser excluída. Serão aceitas publicações que descrevam pelo menos provas de conceito e/ou relatos de experiência na academia ou na indústria.

A seleção dos estudos será feita em 2 etapas:

i) Seleção e catalogação preliminar dos dados coletados: a seleção preliminar das publicações será feita a partir da identificação dos artigos publicados nos anais das conferências no escopo do estudo. Apenas as publicações referentes ao evento principal (exclui-se, por exemplo, eventos paralelos, concursos e workshops de teses e dissertações, keynotes speeches, resumos de mini-cursos etc.) serão consideradas. Cada publicação será catalogada em um banco de dados criado especificamente para este fim e armazenada em um repositório para análise posterior.

ii) Seleção dos dados relevantes - aplicação do filtro: A seleção preliminar não garante que todo o material coletado seja útil no contexto da pesquisa, dessa forma, deve ser feita uma análise das publicações selecionadas e aplicados os seguintes critérios de inclusão (CI) e de exclusão (CE): $C E 1$ - Serão excluídos do estudo todas as publica- 
ções que não citam o MPS.BR nem o CMMI; CI 1 - Serão selecionadas para o estudo as publicações que mencionam o MPS.BR e/ou o CMMI; e CI 2 - Serão selecionadas para o estudo as publicações em que haja indícios de utilização do MPS.BR e/ou o CMMI.

\subsection{Procedimentos para Extração dos Dados}

Seleção e catalogação preliminar dos dados coletados: Deve-se armazenar as referências completas selecionadas a partir da fonte consultada no repositório de dados.

Seleção dos dados relevantes das publicações: Cada referência deve ser examinada com o objetivo de ser submetida aos critérios de seleção dos filtros identificados. Os dados que atenderem aos critérios de seleção deverão ser identificados apropriadamente.

Extração de Dados: Os dados extraídos das publicações selecionadas deverão ser armazenados em um banco de dados e devem conter as informações apresentadas nas Figuras 1a e 1b.

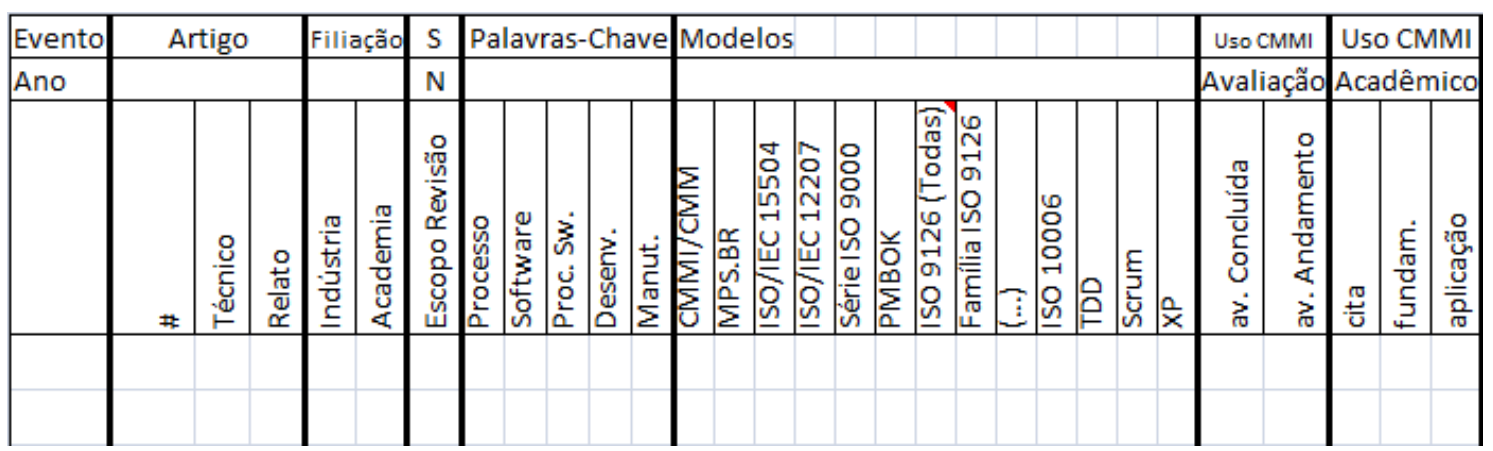

Figura 1a - Formulário de Coleta de Dados (Parte I)

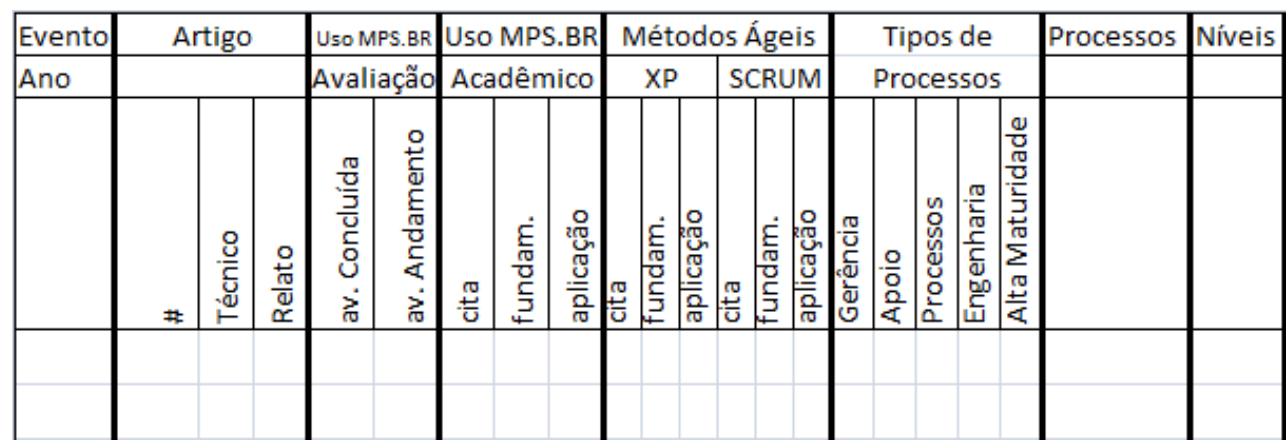

Figura 1b - Formulário de Coleta de Dados (Parte II)

Devem ser informados: o nome e ano do evento considerado, a identificação do artigo (número da página inicial ou número do artigo, se artigo técnico ou relato de experiência), filiação dos autores (academia e/ou indústria), se o artigo faz parte ou não do escopo da revisão sistemática, as palavras-chave identificadas no resumo e/ou abstract, referências ao MPS.BR, CMMI e outros métodos, normas, modelos e padrões de referência são citados pelas publicações selecionadas, detalhamento das menções ao CMMI, MPS.BR, Scrum e XP, tipos dos processos mencionados, processos mencionados e níveis de maturidade mencionados quando há menção à avaliação de empresas.

São considerados relatos de experiência os trabalhos publicados em trilhas específicas para relatos de experiência. São considerados trabalhos técnicos aqueles não considerados como sendo relatos de experiências. 
Para a caracterização do tipo de citação do CMMI e MPS.BR, indica-se, também, se há menção a avaliações oficiais concluídas ou em preparação e se os modelos são citados, utilizados como parte da fundamentação do trabalho ou há indícios que tenham sido de fato utilizados.

É considerado fundamentação quando se propõe o alinhamento do tópico de pesquisa do artigo técnico ao modelo de referência do MPS.BR ou do CMMI, quando se apresenta relatos de experiência envolvendo a obtenção de um nível de maturidade, quando cita-se exemplo de itens requeridos pelos modelos e que são atendidas pela abordagem descrita no artigo, quando se diz que o MPS.BR ou o CMMI são usados como base para definirem um processo. Quando o artigo cita o MPS.BR e o CMMI e depois justifica a abordagem técnica em relação a modelos de maturidade, considera-se como fundamentação tanto pelo MPS.BR quanto pelo CMMI. Só é caracterizado "aplicação" em artigos técnicos quando há indícios que a abordagem realmente foi aplicada em empresas avaliadas em um processo de avaliação, não basta apenas citar uma empresa avaliada. No entanto, em artigos de relatos de experiência, basta citar a ocorrência de uma avaliação.

Identificar uma avaliação como sendo concluída não significa que o artigo comenta sobre avaliação, pode apenas levar a crer que tenha havido uma avaliação oficial.

Para a filiação é considerada a menção ao órgão/instituição/universidade explicitamente no cabeçalho do artigo. A participação da academia se configura se algum dos autores está filiado a pelo menos uma instituição de pesquisa. A participação da indústria se configura se algum dos autores está filiado a pelo menos uma organização que não seja uma instituição de pesquisa. Apenas a inclusão de um e-mail dos autores que possa ser considerado de uma empresa não caracteriza filiação a ela. Empresas de consultoria ligadas ou não a universidades e a departamentos de pesquisa e desenvolvimento $(\mathrm{P} \& \mathrm{D})$ de empresas configuram filiação à indústria e não à academia.

Os possíveis tipos de processo são: gerência, apoio, definição/gerência de processos, engenharia e ou de alta maturidade (referente aos níveis B e A do MR-MPS e 4 e 5 do CMMI).

Devido ao espaço disponível neste artigo, foram suprimidos alguns modelos/normas referenciados da Figura 1a, a lista completa é composta por: CMMI/CMM, MPS.BR, ISO/IEC 15504, ISO/IEC 12207, Série ISO 9000, PMBOK, ISO 9126 (Todas), RUP, TMM, SWEBOK, ISO 21827, ISO 15408, ISO 17799, ISO 15939, ISO/IEC TR 16326, IEEE Std 1062, IEE Std 1540, ISO/DIS 9241, ISO/IEC 12119, ISO/IEC 14598, IEEE 830, AS/NSZ 4360, ISO 14001, IEEE 610, IEEE 829, IEEE 1219 ， IEEE 1471, IEEE 1061, ISO 10015, ISO 19011, QIP, ITIL, OpenUP, COBIT, ISO/IEC 14102, IDEAL, ISO 31000, ISO/IEC 14764, ISO/IEC 16085, IEEE 1028, IEEE 1044, PSP, ISO 10006, TDD, Scrum e XP.

Algumas considerações devem ser feitas sobre os modelos descritos acima: (i) as menções aos modelos podem ser feitas a partir do seu nome diretamente no texto ou a presença de uma referência a ele; (ii) algumas normas podem ser identificadas indistintamente a partir da sua versão em inglês ou a tradução em português; (iii) são consideradas menções válidas ao MPS.BR variações em torno de MPS.BR e MR-MPS, por exemplo: MR-MPS, mps.BR, mpsBR, mps BR, MR mps, MR mps Br, mps-BR etc.; (iv) são também consideradas menções válidas ao CMMI os termos CMM (sua versão anterior) e CMMI-DEV, CMMI-AQU, CMMI-SRV (denominações específicas do mo- 
delo para, respectivamente, Desenvolvimento de Software, Aquisição e Serviços); (v) para a ISO 9126 são consideradas menções a todas as partes da norma e também à sua equivalente nacional (ABNT NBR 13596) e a sua evolução (ISO 25000).

Sumarização dos resultados: Os resultados serão tabulados. Nenhuma meta-análise será realizada.

\subsection{Procedimentos para Análise}

Análise Quantitativa: A análise quantitativa dar-se-á pela extração direta dos dados a partir do banco de dados com os registros dos achados. A análise quantitativa consiste em fornecer: (i) número de publicações selecionadas para fazerem parte do estudo; (ii) número de citações encontradas nas publicações selecionadas para fazerem parte do estudo.

Análise Qualitativa: A análise qualitativa deverá utilizar como base, os dados quantitativos e realizar considerações com o intuito de discutir os achados com relação às questões de pesquisa declaradas.

\section{Execução de Rodada Piloto para Definição do Protocolo}

Uma das etapas mais importantes da condução de um estudo baseado em revisão sistemática é a execução de rodadas piloto. A partir destas rodadas piloto é possível avaliar a versão inicial do protocolo definido, refinando-o de forma a garantir que o planejamento do estudo tenha sido feito adequadamente.

Neste momento, alguns itens importantes de serem revistos são (i) a identificação das fontes de pesquisa, (ii) a identificação de publicações de controle, (iii) a definição do período de buscas, (iv) as palavras chave que serão utilizadas para a identificação das publicações selecionadas no estudo, (v) o método de buscas, (vi) a definição e refinamento de questões de pesquisa, (vii) a definição dos filtros utilizados para a seleção das publicações do estudo e critérios de inclusão e exclusão associados, e (viii) a definição do formulário de coleta de dados das publicações selecionadas pelo estudo.

\subsection{Identificação das Fontes de Pesquisa}

Como o foco deste estudo é na influência do MPS.BR [SOFTEX 2009] na pesquisa em Qualidade de Software no Brasil, foi definido que o escopo das buscas seria composto pelos anais do Simpósio Brasileiro de Qualidade de Software, principal evento da área no país. Outros eventos, como o Simpósio Brasileiro de Engenharia de Software (SBES) e o Simpósio Brasileiro de Sistemas de Informação (SBSI) foram considerados, porém descartados devido ao foco mais abrangente.

\subsection{Publicações de Controle e Período de Buscas}

Para este estudo, desde o início foi pensado que não haveria, de fato, uma ou mais publicações de controle, mas seriam consideradas edições específicas do SBQS para análise. Apesar de não ter sido a primeira publicação a mencionar o MPS.BR (há publicações anteriores no QUATIC e CLEI), foi identificado como uma das publicações mais importantes do período inicial de implantação um artigo publicado pela Equipe Técnica do Modelo (ETM) no SBQS de 2005 em Porto Alegre intitulado "Modelo de Referência e Método de Avaliação para Melhoria de Processos de Software - versão 1.0 (MR-MPS e MA-MPS)" [WEBER et al. 2005]. O SBQS surgiu em 2002 a partir da evolução do Workshop de Qualidade de Software (WoQS). O MPS.BR começou a surgir em 2003 e 
as primeiras avaliações piloto foram executadas em 2005. Este artigo representa um momento inicial de consolidação do Programa MPS.BR e de seus principais componentes: o Modelo de Referência (MPS.BR), o Método de Avaliação (MA-MPS) e o Modelo de Negócio (MN-MPS). A aceitação do artigo (considerado o melhor trabalho técnico daquela edição do evento) também representa um reconhecimento da comunidade científica pelo trabalho realizado. A Tabela 1 apresenta os principais marcos.

Tabela 1 - Principais Marcos

\begin{tabular}{|c|l|}
\hline Ano & \multicolumn{1}{|c|}{ Evento } \\
\hline 2002 & Primeira edição do SBQS \\
\hline 2003 & Criação do MPS.BR em reunião no MCT em 11/12/2003, em Brasília-DF \\
\hline 2004 & Criação do MN-MPS em 15/04/2004, em Brasília-DF \\
\hline 2005 & $\begin{array}{l}\text { Criação do MR-MPS (Guia Geral 1.0) e do MA-MPS (Guia de Avaliação 1.0, versão piloto) em } \\
\text { maio/2005, artigo do MPSBR no SBQS 2005 (junho/2005), primeiras avaliações pilo- } \\
\text { to (iniciadas em setembro/2005, com base na versão piloto do Guia de Avaliação 1.0) }\end{array}$ \\
\hline 2006 & Publicação do MR-MPS (Guia Geral 1.1) e do MA-MPS (Guia de Avaliação 1.0) em maio/2006 \\
\hline 2009 & $\begin{array}{l}\text { Publicação do MR-MPS (Guia Geral versão 2009) e do MA-MPS (Guia de Avaliação versão } \\
\text { 2009) em junho/2009 }\end{array}$ \\
\hline 2010 & Edição mais recente do SBQS \\
\hline
\end{tabular}

A partir da identificação deste artigo, foi definido que seriam utilizados na rodada piloto todos os artigos da edição de 2005 do SBQS e também das edições ocorridas dois anos antes e após (ou seja, 2003 e 2007). Dessa forma, esperava-se identificar se as citações ao MPS.BR tinham, de fato, se tornado mais freqüentes e, também, possibilitar uma comparação com as citações feitas ao CMM/CMMI (um modelo de maturidade mais antigo e, de certa forma, precursor do MPS.BR e também bem difundido no país).

Ao final desta rodada, apesar de não haver ainda dados conclusivos, pôde-se perceber que em 2007 já havia um número de citações ao MPS.BR próximo ao do CMMI. Além disso, foi verificado que em 2003 não havia citações ao MPS.BR e em 2005 havia um número bastante reduzido, conforme esperado. Dessa forma, foi concluído que a continuação dos esforços na condução do estudo era justificável.

Apesar de não haver referências ao MPS.BR em 2003 (posteriormente verificouse que também não havia em 2002 e 2004, como era de se esperar), todas as edições do SBQS foram consideradas relevantes para que pudesse fazer comparações dos resultados obtidos em relação ao MPS.BR com aqueles relacionados ao CMMI. Dessa forma, o período de buscas foi definido, então, como sendo todo o de existência do SBQS (ou seja, de 2002 até os dias de hoje).

\subsection{Palavras-chave}

Em um primeiro momento foram consideradas as seguintes palavras-chave: processo, software, processo de software, desenvolvimento e manutenção. A rigor, "processo de software" poderia ter sido excluída, pois todos os artigos que as contivessem também seriam identificados por "processo" e "software". Porém, durante a rodada piloto era desejado avaliar se a adoção de "processo de software" em vez de "processo" e "software" traria menos falso-positivos. Ao final da rodada piloto foi identificado um artigo que, apesar de não ter nenhuma das palavras-chave acima deveria estar no escopo do estudo. Foi decidido, então, que todos os artigos estariam contidos no escopo inicial da revisão sistemática, no entanto, coletar-se-ia a presença ou não das palavras-chave nas publicações para possíveis análises futuras.

Para os artigos em inglês e espanhol foram utilizados as traduções das palavras- 
chave nestes idiomas. Para os artigos em português foi considerada também a presença das traduções em inglês nos abstracts. Alternativamente, foram consideradas também as seguintes variações das palavras-chave: processos de software, processo do software e processos.

\subsection{Método de Buscas}

Os anais do SBQS não estão disponíveis em nenhuma biblioteca digital com mecanismos de indexação e busca eletrônica, desta forma, só foram realizadas buscas manuais, tanto nos anais em papel quanto em mídia eletrônica.

Para os artigos impressos, a busca pelas palavras-chave, e referências aos modelos considerados, se deu manualmente a partir da leitura do texto completo. Procurou-se identificar as menções aos modelos e normas tanto no conteúdo texto quanto nas referências bibliográficas. Para os anais em mídia eletrônica, foram utilizados os mecanismos de buscas de palavras do leitor de PDF utilizado, a busca manual pela leitura do texto e, também, uma macro do MS-Word construída para buscar e destacar no texto todas as palavras pesquisadas.

\subsection{Questões de Pesquisa}

Em um primeiro momento foi definida a questão de pesquisa principal (QP 1), que se manteve ao longo do estudo. Houve apenas modificações na sua formalização, sem alterações de conteúdo.

Apesar de o foco do estudo ser na influência do MPS.BR, as questões de pesquisa mencionam tanto o MPS.BR quanto o CMMI para que seja possível realizar comparações sobre a adoção de ambos os modelos (QS 1), aumentando, assim, as possibilidades de análise dos resultados. Durante a execução da rodada piloto, com a leitura dos artigos, foram sendo definidas as questões secundárias que procuram detalhar como e em que grau é observada a influência do MPS.BR e do CMMI presente nos artigos (QS 3, QS 4, QS 5 e QS 6). A questão referente a outros métodos, normas, modelos e padrões de referência (QS 2) foi criada a partir da observação de citações semelhantes em um estudo baseado em revisão sistemática sobre o uso de apoio ferramental em iniciativas de melhoria de processos de software [SANTOS 2008].

Por fim, tem-se percebido um crescente interesse em métodos ágeis por empresas de software no Brasil. Foram identificadas menções a Extreme Programming (XP) em 2003 e 2007 durante a rodada piloto (apenas 1 artigo em cada evento, não sendo identificadas menções em 2005). Assim, decidiu-se aproveitar o esforço que já seria realizado de analisar todas as publicações e incluir uma questão de pesquisa (QS 7) específica às citações de dois dos métodos ágeis mais difundidos, o XP [BECK ANDRES 2005] e o Scrum [ScrumAlliance 2011]. O esforço adicional para pesquisar também por estes dois métodos não foi considerado representativo em relação ao esforço da pesquisa como um todo, pois o universo total pesquisado foi de 47 modelos/normas.

\subsection{Filtros e Critérios de Inclusão e Exclusão}

Em um primeiro momento pensou-se em utilizar as palavras-chave como o primeiro filtro para selecionar as publicações a serem incluídas no escopo desta pesquisa, no entanto, verificou-se que a melhor alternativa era analisar todos os artigos identificados indistintamente e procurar no texto completo deles a presença de menções ao MPS.BR (e MR-MPS) e CMMI (e CMM). Isso foi feito para não eliminar nenhum potencial arti- 
go para o estudo antes da sua completa análise. Os critérios de inclusão e exclusão foram definidos para refletir essa realidade. Durante a coleta dos dados será feita a análise se os artigos apenas mencionam/citam, se fundamentam ou descrevem a aplicação prática do uso dos modelos em questão.

\subsection{Formulário de Coleta de Dados}

O formulário de coleta de dados teve sua primeira versão elaborada antes do início da execução da rodada piloto. Ao longo da leitura dos artigos, à medida que as questões secundárias de pesquisa eram formuladas e revisadas, o formulário foi sofrendo modificações e ajustes. Além da necessidade de responder às questões de pesquisa, foram incluídos no formulário campos para a caracterização dos artigos, incluindo: nome e ano do evento considerado, identificação do artigo (que pode ser o número a página inicial, quando os anais foram consultados a partir do texto completo dos anais impressos ou em meio eletrônico, ou o número do artigo, quando eram consultados os artigos em meio eletrônico de forma isolada dos anais), o tipo de artigo (se trabalho técnico ou relato de experiência) e a filiação dos autores (se oriundos da academia ou da industria).

Ao final da rodada piloto, foi definida a estrutura padrão do formulário de forma a possibilitar a coleta de todas as informações relacionadas às questões de pesquisa formuladas. Ao longo da execução da pesquisa (fora da rodada piloto) foram feitas adequações de forma a reduzir redundâncias, facilitar seu preenchimento e, também, incluir referencias a outros modelos não identificados anteriormente. Estas alterações não implicaram em necessidade de analisar novamente as publicações já analisadas. A versão final do formulário pode ser vista na Figura 1. A lista inicial de modelos considerados foi composta por CMMI / CMM, MR-MPS / MPS.BR, ISO/IEC 15504, ISO/IEC 12207, Série ISO 9000, PMBOK, ISO 9126 e ISO 25000. Posteriormente esta lista evoluiu para a sua configuração atual.

Os dados coletados foram anotados em um formulário impresso, para facilitar e aumentar a velocidade da coleta dos dados. Depois, foram transcritos para planilhas Excel e, então, importados para uma base de dados no Access para consolidação e geração dos gráficos. Procedimentos de checagem cruzada dos dados foram adotados para avaliar a transposição dos dados coletados em papel para o meio eletrônico. Em caso de possível divergência, os artigos eram reavaliados e ajustes, caso necessário, realizados.

\subsection{Ameaças à Validade do Estudo}

Ameaça à validade interna: São eventos não controlados que podem produzir distorções no resultado esperado. Parte da busca nos artigos foi feita manualmente, que por ser uma atividade repetitiva e cansativa, pode ter ocasionada possíveis falhas na catalogação dos resultados. Além disso, toda a coleta e análise dos dados foram feitas por um único pesquisador, que também é membro da Equipe Técnica do MPS.BR.

Ameaça à validade externa: As ameaças deste tipo prejudicam a generalização dos resultados do estudo. Dentre as principais limitações do estudo está o fato de considerar para o escopo da pesquisa apenas as publicações do Simpósio Brasileiro de Qualidade de Software (SBQS). Além disso, não foi feita uma análise qualitativa do uso dos elementos do MPS.BR e/ou o CMMI quando estes foram utilizados como justificativa/fundamentação da pesquisa realizada.

Ameaça à validade de construção: São eventos que podem prejudicar a medição correta no estudo. Como a medida (contagem das publicações citadas) utilizada foram a- 
presentada, não foram identificadas ameaças deste tipo.

Ameaça à validade de conclusão: Estas ameaças prejudicam o estabelecimento de relacionamentos estatísticos. Neste estudo não foram realizados testes estatísticos, assim, os resultados não poderão ser considerados conclusivos.

\section{Principais Resultados Obtidos}

Esta seção descreve os principais resultados decorrentes da execução do protocolo do estudo baseado em revisão sistemática descrito na Seção 2. Foram analisados os anais do SBQS no período compreendido entre 2002 e 2010, inclusive. Apenas um pesquisador analisou todas as publicações, dessa forma, não houve diferença no julgamento e análise das publicações. Foram considerados como estando dentro do escopo desta revisão sistemática todos os artigos que mencionaram pelo menos o CMMI e o MPS.BR, conforme descrito anteriormente. Dos 252 artigos analisados, 175 fizeram parte do escopo do estudo, conforme mostra a Figura 2. Para cada ano são informados quantos artigos foram publicados nos anais do SBQS e quantos foram considerados como dentro do escopo do estudo. Pode-se perceber que após 2005, mais de 50\% dos artigos mencionam pelo menos o CMMI ou o MPS.BR, sendo que em 2009 a taxa ficou em $83 \%$.

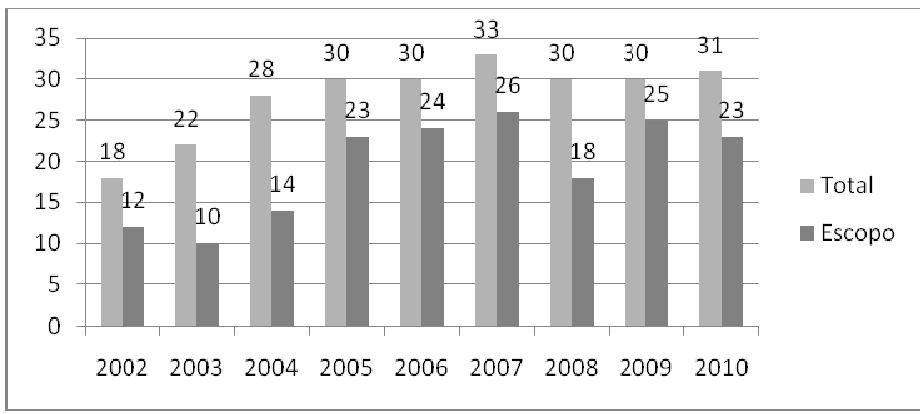

Figura 2 - Publicações no Escopo da Revisão Sistemática

A Figura 3 apresenta a distribuição dos artigos selecionados para o estudo de acordo com a filiação dos autores: todos os autores ligados apenas à indústria, todos os autores ligados apenas à academia e presença de autores oriundos tanto da academia quanto da indústria. Pode-se perceber que a filiação à academia e à indústria+academia oscila em torno dos mesmos valores anualmente.

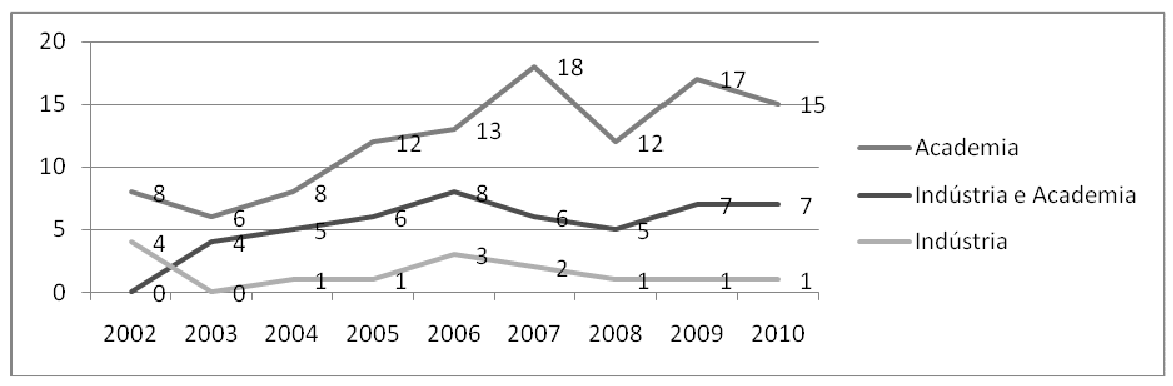

Figura 3 - Filiação dos Autores dos Artigos Selecionados

A Figura 4 apresenta o número de vezes que cada método, norma, modelo e padrão de referência é citado pelas publicações selecionadas (ver questão secundária de pesquisa QS 2). Pode-se perceber que o número de citações do CMMI é quase o dobro do MPS.BR, considerando-se o período total desde 2002. Esta diferença tem diminuído com o passar do tempo, como será visto a seguir.

A Figura 5 analisa a citação dos 5 principais modelos (CMMI, MPS.BR, I- 
SO/IEC 15504, ISO/IEC 12207 e ISO 9000) ao longo dos anos. Pode-se perceber que o número de citações ao MPS.BR está se aproximando do número de citações ao CMMI, tendo ultrapassado-o em 2010. Não se pode perceber um padrão no gráfico, porém há uma tendência de que o MPS.BR estejam se tornando tão ou mais influente nas pesquisas ao longo do tempo (ver QS 1). A relação do número de citações entre o MPS.BR e o CMMI é próxima, talvez devido às similaridades entre eles. O fato de a ISO/IEC 12207 e a ISO/IEC 15504 serem muito citadas decorre, muitas vezes, do fato de elas serem utilizadas como base para a definição do MR-MPS e, assim, na revisão da literatura dos artigos serem citadas em conjunto ao MR-MPS.

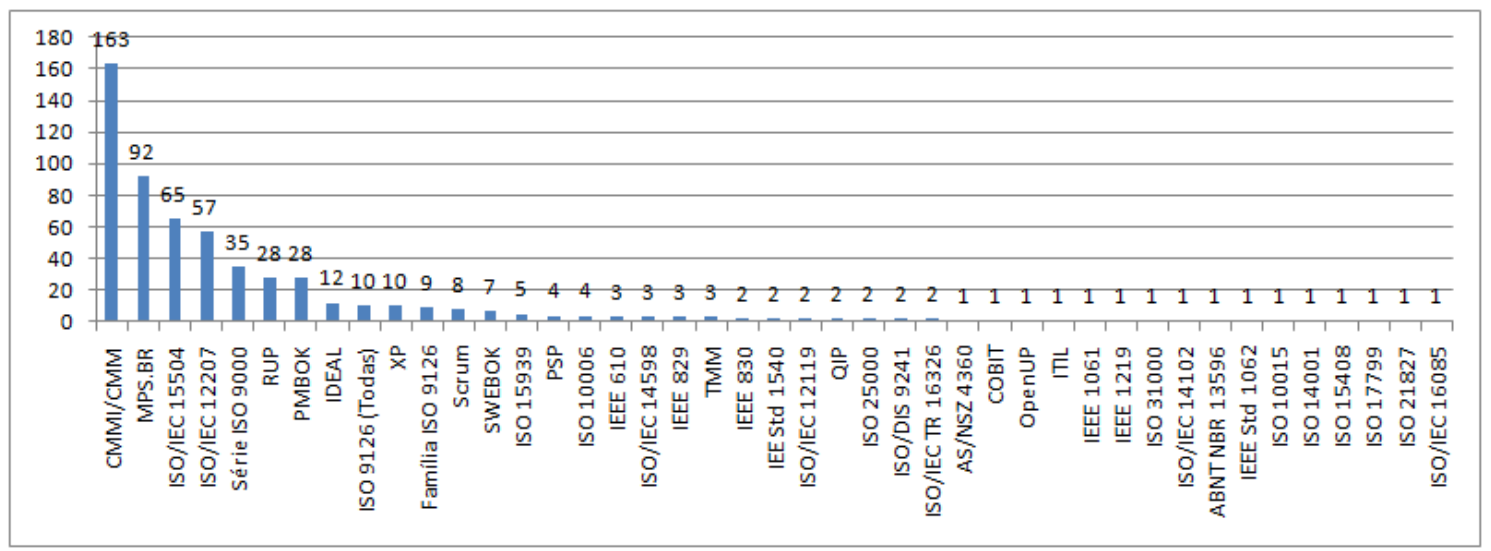

Figura 4 - Menções a Modelos de Referência no Escopo da Revisão Sistemática

De qualquer forma, a partir do ano de criação do MPS.BR também aumentou-se o número de publicações que referenciam o CMMI, de alguma forma parece que a criação do MPS.BR tenha influenciado os pesquisadores a prestarem maior atenção aos itens preconizados pelos modelos de maturidade (seja o CMMI, seja o MPS.BR).

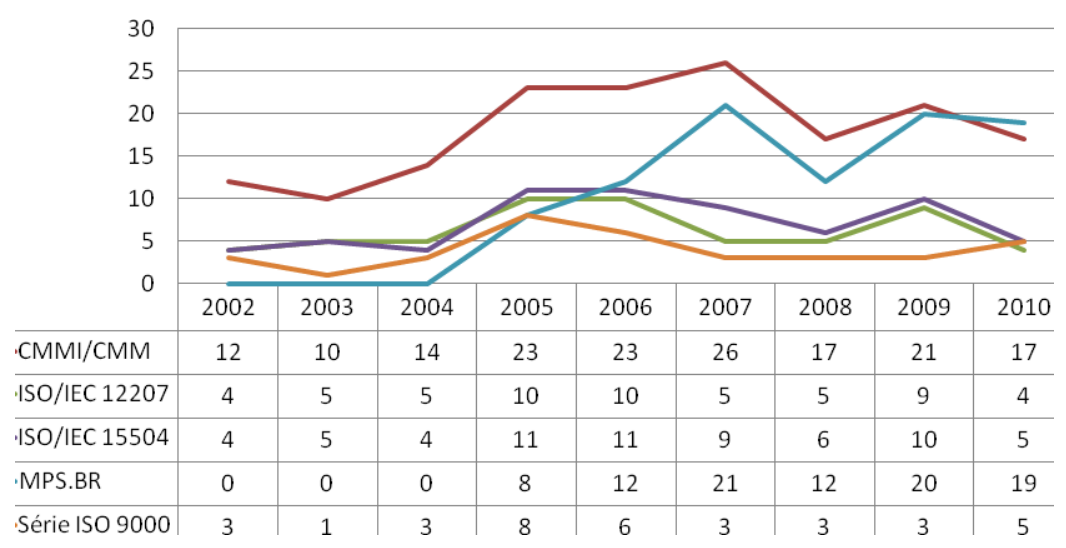

Figura 5 - Menções aos Modelos de Referência mais Citados

As Figuras 6 e 7 apresentam, respectivamente, a distribuição do tipo de citação que é feita em relação ao MPS.BR e ao CMMI (ver QS 1, QS 3 e QS 4). Para cada modelo é indicado quantas vezes ele é citado, utilizado para fundamentar a pesquisa e é evidenciada uma aplicação prática dele. Note que apesar do item "aplicação" aparecer na parte inferior dos gráficos ele é mais relevante que apenas a "citação" do modelo. Assim, em ordem de relevância: um modelo pode ser utilizado como citação, como parte da fundamentação e evidenciado sua utilização na pesquisa. Todos os artigos que compõem o item "aplicação" também são contados no item "fundamentação", da mesma forma, todos os artigos contados em "fundamentação" também são contados no item 
“citação". As duas séries só podem ser comparadas a partir de 2005, pois antes não havia citações ao MPS.BR. Novamente os valores continuam oscilando ao longo do tempo, porém em alguns anos, o número de artigos que utilizam o MPS.BR como parte da fundamentação ou aplicação é superior aos que utilizam o CMMI (anos 2008 e 2009).

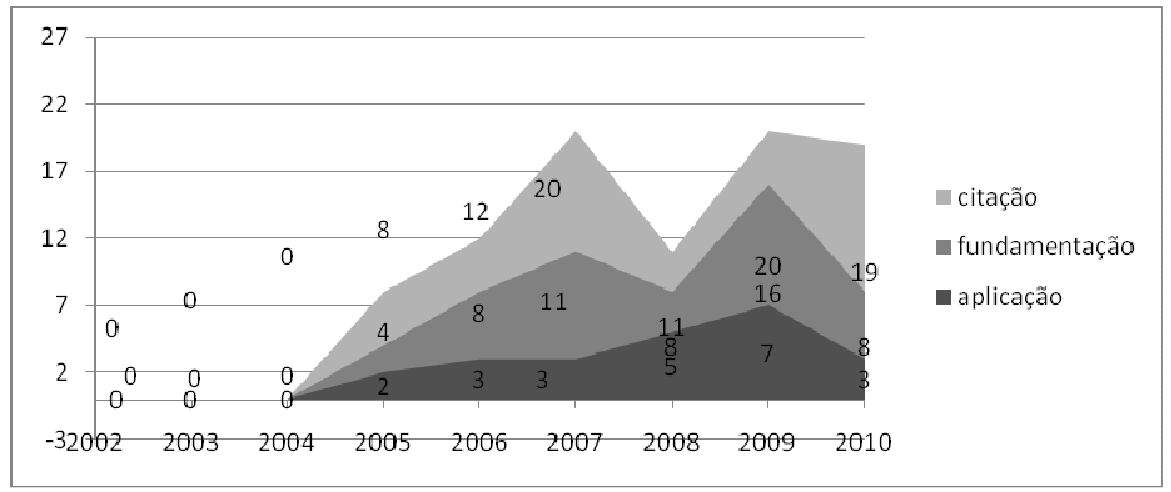

Figura 6 - Citações ao MPS.BR nos artigos

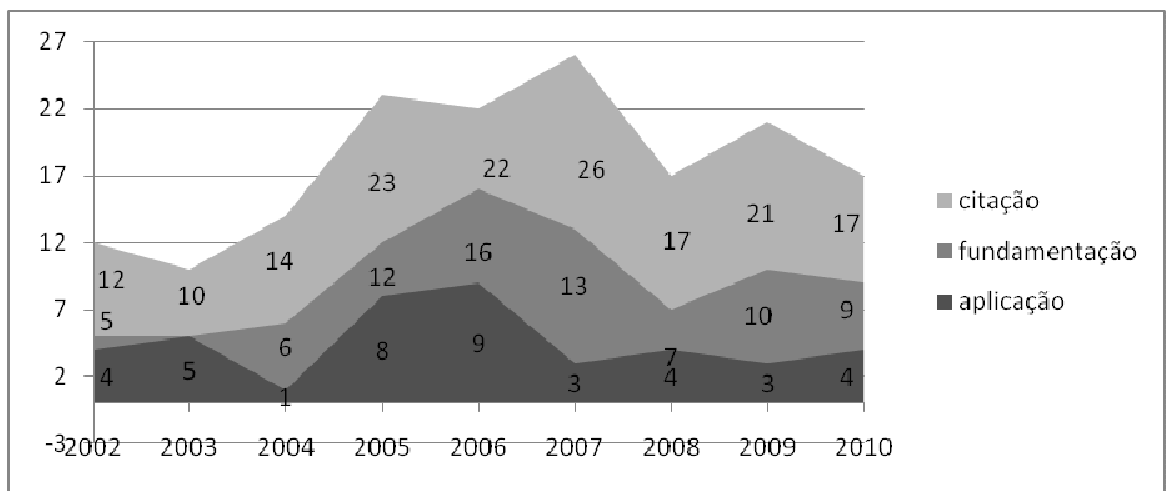

Figura 7 - Citações ao CMMI nos artigos

As Figuras 8a e 8b procuram responder à questão secundária QS 5. A Figura 8a exibe o número de vezes que processos/aspectos (de uma fora geral) gerenciais, de apoio, de gerência de processos e de alta maturidade foram citados nos artigos selecionados. $\mathrm{O}$ grande número de artigos relacionados à alta maturidade merece atenção porque são poucas as organizações de software no Brasil que são avaliadas nos níveis B e A do MR-MPS ou 4 e 5 do CMMI, porém uma boa parte dos artigos onde as citações foram identificadas são relatos de experiência. A Figura 8b apresenta os processos citados explicitamente (as siglas exibidas são aquelas do MR-MPS, mesmo quando o artigo citou o nome do processo equivalente do CMMI). O processo com maior número de citações é o de Gerência de Requisitos (7). Note que nenhum processo dos níveis de alta maturidade foi citado explicitamente.

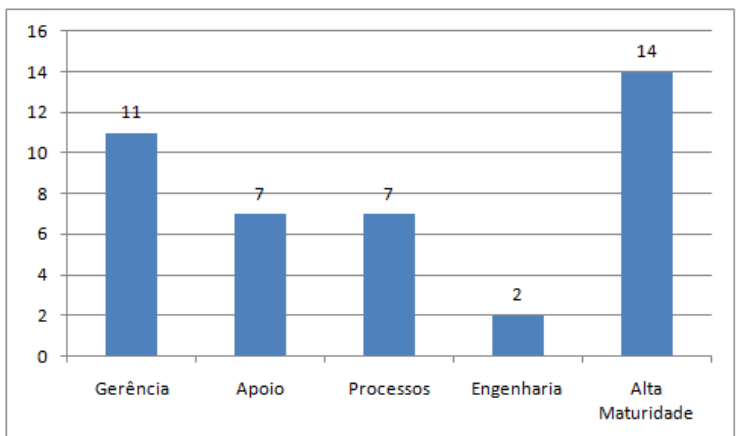

Figura 8a - Tipos de Processo Mencionados

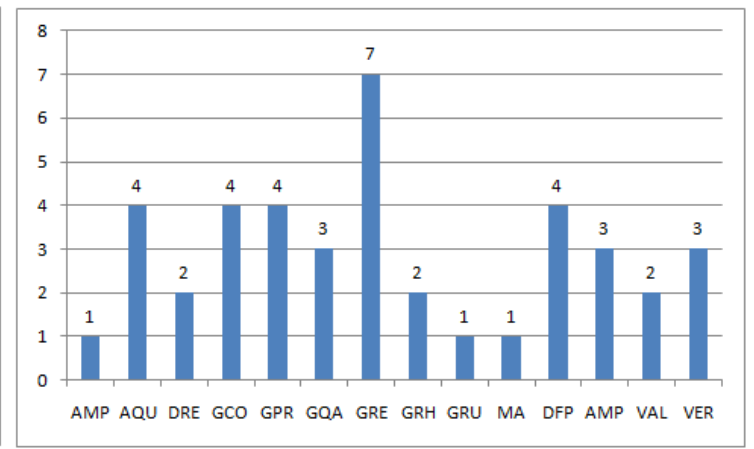

Figura 8b - Processos Mencionados 
As avaliações CMMI ou MPS.BR citadas (ver QS 6) foram: Nível G do MRMPS (8 vezes), Nível F do MR-MPS ou Nível 2 do CMMI ou CMM (35 vezes), Nível E do MR-MPS (6 vezes), Nível C do MR-MPS ou Nível 3 do CMMI ou CMM (23 vezes) e Nível 5 do CMMI ou CMM (3 vezes). Estas avaliações não são contadas unicamente, ou seja, podem ser contadas duas vezes se são citadas em dois artigos diferentes, por exemplo. Estes valores, no entanto, não são proporcionais ao número de avaliações MPS já realizadas no país segundo a base de dados SOFTEX (disponível em www.softex.br/mpsbr), de setembro de 2005 até 11 de abril 2011, foram publicadas 280 avaliações MPS, das quais: $\mathrm{G}=173$ (62\%), $\mathrm{F}=80$ (29\%), $\mathrm{E}=9$ (3\%), $\mathrm{D}=1(-\%), \mathrm{C}=$ $10(4 \%), \mathrm{B}=0(0 \%)$ e $\mathrm{A}=7(2 \%)$.

As Figuras 9a e 9b apresentam a distribuição do tipo de citação que é feita em relação ao XP e ao Scrum (ver QS 7), respectivamente. O que pode-se perceber é que o o uso do XP e do Scrum nas pesquisas relacionadas a Qualidade de Software em conjunto com o MR-MPS e o CMMI ainda é incipiente, atingindo o máximo em 2010, mesmo assim com pouquíssimas citações (2 para o XP e 3 para o Scrum). A única outra citação a método ágil identificado nas publicações dentro do escopo da pesquisa foi o TDD (Test Driven Development), porém não aparece listado na Figura 9 porque a publicação onde a citação foi feita não foi selecionada para o escopo final da revisão sistemática (ou seja, não atendeu aos critérios de inclusão definidos).

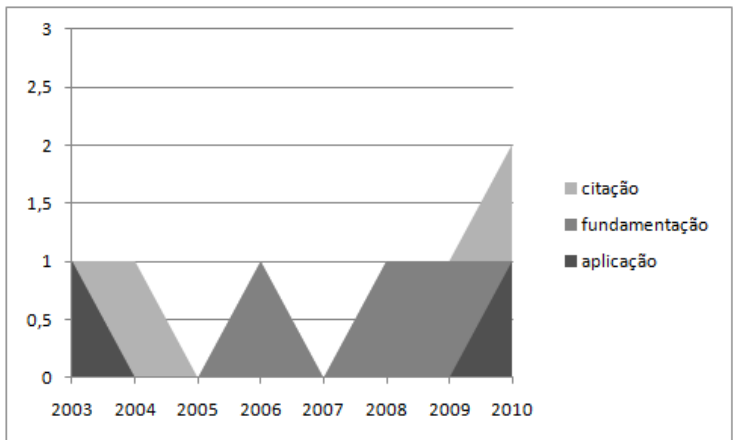

Figura 9a - Citações ao XP nos Artigos

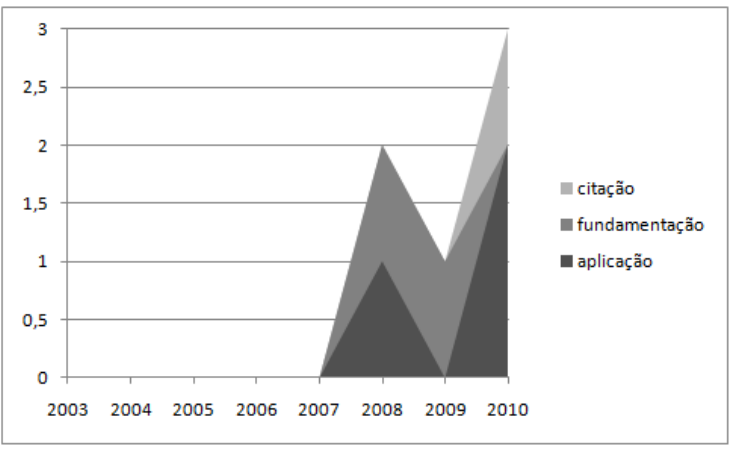

Figura 9b - Citações ao Scrum nos Artigos

\section{Considerações Finais}

Este artigo apresentou o planejamento e o resultado de um estudo baseado em revisão sistemática que objetivou avaliar o impacto da criação do Programa MPS.BR e o seu Modelo de Referência (MR-MPS) na pesquisa em Qualidade de Software no país. Foram analisadas todas as publicações de todas as edições do Simpósio Brasileiro de Qualidade de Software (SBQS). Em relação à questão de pesquisa principal deste trabalho ("O Programa MPS.BR e o seu Modelo de Referência (MR-MPS) estão influenciando a pesquisa em Qualidade de Software no Brasil?"), ainda é cedo para se afirmar com certeza. No entanto, há indícios que desde que o MPS.BR foi criado houve um aumento no número de publicações que se fundamentam em algum aspecto do seu Modelo de Referência (MR-MPS) para fundamentar algum tópico de pesquisa e, também, pode-se perceber o aumento do número de relatos de experiência sobre o MPS.BR, constituindo assim uma evidência indireta da transferência de conhecimento entre a academia e a indústria em decorrência da utilização do modelo. Outro fator a se destacar é a possível tendência dos últimos anos de haver mais artigos publicados mencionando a aplicação de aspectos do MR-MPS na pesquisa em Qualidade de Software do que o CMMI, tradicional e mais antigo modelo de maturidade. 
Como próximo trabalho espera-se a atualização dos resultados desta pesquisa a partir da avaliação dos artigos a serem publicados no SBQS no futuro. Outro trabalho futuro é o aumento do escopo desta revisão sistemática para incluir também outros eventos nacionais patrocinados pela Sociedade Brasileira de Computação (SBC), como o Simpósio Brasileiro de Engenharia de Software (SBES) e o Simpósio Brasileiro de Sistemas de Informação (SBSI). Além disso, espera-se também revisar o protocolo e reexecutar o estudo visando realizar uma análise qualitativa do uso do MPS.BR na pesquias relacionada a Qualidade de Software e, também, avaliar, se possível, o que mudou no desenvolvimento de software no Brasil com o MPS.BR.

\section{Agradecimentos}

$\mathrm{O}$ autor agradece à FAPERJ pelo auxílio financeiro.

\section{Referências Bibliográficas}

BECK, K.; ANDRES, C. (2005) "Extreme Programming Explained: Embrace Change", 2 ed. Upper Saddle River: Addison-Wesley.

BIOLCHINI, J., MIAN, P.G., NATALI, A.C., et al., 2005, Systematic Review in Software Engineering: Relevance and Utility, Technical Report ES-679/05, Programa de Engenharia de Sistemas e Computação, Universidade Federal do Rio de Janeiro, Rio de Janeiro.

KALINOWSKI, M., WEBER, K. and TRAVASSOS, G.H. (2008). iMPS: An Experimentation Based Investigation of a Nationwide Software Development Reference Model. ACM/IEEE 2nd International Symposium on Empirical Software Engineering and Measurement. October, 9-10. Kaiserslautern. Germany.

KALINOWSKI, M., SANTOS, G., REINEHR, S., MONTONI, M., ROCHA, A.R., WEBER, K.C., TRAVASSOS, G.H.: MPS.BR: Promovendo a Adoção de Boas Práticas de Engenharia de Software pela Indústria Brasileira. XIII Congresso Iberoamericano de Engenharia de Software (CIBSE), Cuenca, Equador (2010).

KITCHENHAM, B., 2004, Procedures for Performing Systematic Reviews, Technical Report, Departament of Computer Science Keele University, Keele.

MAFRA, S., TRAVASSOS, G.H., 2006, Estudos Primários e Secundários apoiando a busca por Evidência em Engenharia de Software, Relatório Técnico RT-ES 687/06, Programa de Engenharia de Sistemas e Computação, Universidade Federal do Rio de Janeiro, Rio de Janeiro.

SANTOS, G., 2008, Ambientes de Engenharia de Software Orientados à Corporação. Tese de D. Sc., COPPE/UFRJ, Rio de Janeiro, RJ, Brasil.

SANTOS, G.; WEBER; K. C.; ROCHA, A. R. (2009). Software Process Improvement in Brazil: Evolving the MPS Model and Consolidating the MPS.BR Program, XXXV Conferência Latina Americana de Informática (CLEI 2009), Pelotas - RS.

SCRUMALLIANCE (2011). In: http://www.scrumalliance.org/, último acesso em 20/02/2011.

SILVA FILHO, R. C., 2006, Uma Abordagem para Avaliação de Propostas de Melhoria em Processos de Software. Dissertação de M.Sc., COPPE/UFRJ, Rio de Janeiro, RJ, Brasil.

SOFTEX (2009), "MPS.BR: Melhoria de Processo do Software Brasileiro". In: http://www.softex.br/mpsbr/, último acesso em 02/03/2011.

TRAVASSOS, G. H.; KALINOWSKI, M. (2011). iMPS 2010 - Desempenho das Empresas que Adotaram o Modelo MPS de 2008 a 2010. Campinas, SP: SOFTEX, 2011 (ISBN 978-8599334-20-1) (disponível em http://www.softex.br/mpsbr/_livros/ resultado_desempenho.asp último acesso em 18/04/2011).

WEBER, K. C., ARAÚJO, E., MACHADO, C. A. F., SCALET, D., SAlviANO, C. F., ROCHA, A. R. (2005). Modelo de Referência e Método de Avaliação para Melhoria de Processos de Software - versão 1.0 (MR-MPS e MA-MPS), IV Simpósio Brasileiro de Qualidade de Software (SBQS 2005). Porto Alegre, RS. 